\title{
DÉCONFINER LES CONSCIENCES PAR LA SYSTÉMIQUE
}

\author{
Author(s) / Auteur(s) : \\ Richard VITRAC \\ Electronics Engineer, ENSERG \\ richard.vitrac@gmail.com
}

\begin{abstract}
Résumé :
The Covid-19 pandemic has affected all of humanity. It was the first time that virtually all human beings on earth felt concerned by a particular event: a Chinese allegedly ate pangolin infected with a virus, causing an epidemic that has spread to all mankind.

In the case of covid 19, virtually all humans have been informed by television and the internet. This made them stand together beyond any language, race or religion. However, this shared solidarity was based on the fear of illness and death. There was nothing to counterbalance this atavistic fear, other than, for some, a faith in a particular religion.

On the contrary, systemic sciences, through Cognitive and Systemic Relativity (CSR), shows that fundamentally, our consciousness is immortal because it is not subject to time; it is inspatiotemporal or eternal. It only depends on each one to awaken his social consciousness (his ego) to its dimension of eternity, which corresponds to his EGO which is the Relativist Observer, pilot of the body.

In addition, this individual awakening has the advantage of functioning as a positive pandemic, based on confidence in the source of life which is the Consciousness of Existing, the root of our EGO, and therefore also of our ego.

This positive pandemic is based on a particular functioning which is the systemic entanglement of all the systems on earth since the EGO of all living systems on earth are in communion at all times.

The conscious restoration of this communion of all EGO through trust in our individual EGO sets in motion the healing software of all systems on earth.

This corresponds to the entry of humanity into what we might call universal brotherhood and which Teilhard de Chardin calls the noosphere.
\end{abstract}

\section{Keywords / Mots-clés :}

pandemic, consciousness, Cognitive and Systemic Relativity (CSR), entanglement, Lorentz-Poincaré Formulas

\section{INTRODUCTION}

La pandémie liée à la maladie Covid-19 a pris l'ampleur que l'on constate car elle a été relayée par les moyens modernes de communication. Cela fait que pratiquement chaque être humain sait qu'il risque d'être infecté par le virus. Cela a obligé les dirigeants politiques, économiques et religieux des Etats du monde à se positionner par rapport à cette pandémie. Pour la première fois, l'humanité a fonctionné comme un système géré impliquant tous ses sous-systèmes que sont les êtres humains. Il y avait eu un précédent à la fin de la dernière guerre mondiale, en 1945, lorsque les dirigeants du monde se sont réunis à San Francisco pour signer la Charte des Nations Unies qui a donné naissance à l'ONU, l'Organisation des Nations Unies.

Cependant, dans les deux cas, cette solidarité partagée a été basée sur la peur. C'est-à-dire que dans les deux cas, nous avons été dans une pandémie de la peur.

Or, la systémique, par la relativité cognitive et systémique (RCS), met en évidence que fondamentalement notre conscience est immortelle car elle n'est pas soumise au temps; elle est inspatiotemporelle ou éternelle.

Cela veut dire que, dans le cas de la Covid-19, la "modélisation systémique peut nous aider à tirer parti de cette situation inédite pour nous inciter à décloisonner nos consciences. Cela nous rendrait capables 
d'éviter le choix tentant de la facilité pour mobiliser nos consciences au service des questions qui dérangent. N'oublions jamais notre capacité collective à repenser le monde !" (Claeys, 2020).

Ce texte de l'appel à contribution nous propose de "décloisonner" nos consciences. Cela veut dire qu'il faut envisager la pandémie à partir d'une prise de conscience individuelle de notre place dans l'humanité de la planète terre envisagée comme étant un système vivant, une biosphère ${ }^{1,2}$, et non à partir d'une peur atavique individuelle et collective de la maladie et de la mort. Cela suppose de modéliser le fonctionnement de l'être humain envisagé comme étant un sous-système du système terre.

Cela permettra de transformer la pandémie de Sars-CoV2 en une pandémie positive, basée sur la confiance en la source de la vie qui est la Conscience d'Exister des pilotes de tous les systèmes de l'univers. Cela correspondra à l'entrée de l'humanité dans ce que nous pourrions nommer la fraternité universelle. C'est ce que nous allons voir maintenant.

\section{LA TERRE EN TANT QUE BIOSPHÈRE}

\section{La notion de biosphère à partir de l'intrication systémique}

\section{La vie en tant que manifestation de l'interaction entre les systèmes vivants}

La vie de la planète est caractérisée par l'interaction entre les différentes formes de vie. C'est ce que le biologiste Pierre BRICAGE nomme le fonctionnement "gagnant-gagnant" des systèmes vivants. C'est ainsi que les enfants humains naissent de la fécondation de la femme par l'homme lors des relations sexuelles. Ce processus masculin féminin est général et il peut faire intervenir des systèmes appartenant à des règnes différents. Par exemple, les abeilles ont un rôle essentiel dans la pollinisation des plantes. Une abeille recueille le pollen et le nectar de la fleur. Puis, lorsqu'elle se pose sur une autre fleur, ce nectar est déposé sur le stigmate du pistil (organe de reproduction femelle). C'est grâce à cela que la fécondation est possible et que des graines peuvent se développer.

Cette interaction entre les systèmes biologiques met en évidence l'importance de la biodiversité. Le sommet de la terre de Rio de Janeiro en 1992 a considéré que la préservation de la biodiversité est un des enjeux essentiels du développement durable...

La RCS permet de comprendre comment la biodiversité est fondamentale pour la vie de la planète mais aussi pour la vie humaine.

Curieusement, c'est l'invariance de la vitesse de la lumière qui permet de comprendre la biodiversité et le fait que la terre est une biosphère dans le sens systémique du terme.

\section{Le paradoxe de l'invariance de c à partir de la systémique}

\section{La démonstration de la vitesse de la lumière par Poincaré}

En 1905, peu de temps avant qu'Einstein propose sa théorie de la relativité, le mathématicien Poincaré a prouvé qu'il y avait une vitesse limite dans l'univers qui est la vitesse $\mathrm{c}$ de la lumière.

Pour rendre son raisonnement compréhensible par tous ${ }^{3}$, je le formule d'une façon systémique et non pas mathématique comme l'avait fait Poincaré. Prenons l'exemple d'un avion R qui tourne autour de la terre $\mathrm{T}$ à une vitesse constante. L'avion est un système qui se déplace à une vitesse constante par rapport à la terre qui est un autre système. Poincaré fait l'hypothèse que tous les "passagers" de $\mathrm{R}$ ont leurs montres, mais aussi leurs horloges biologiques, synchronisées sur le rythme propre à l'avion.

\footnotetext{
1 Selon Wikipédia, la biosphère est "le système écologique global auto-entretenu qui intègre tous les êtres vivants et les relations qu'ils tissent entre eux et avec les compartiments que sont la lithosphère (les roches), l'hydrosphère (l'eau) et l'atmosphère (air), dans un métabolisme qui transforme sans cesse la surface de la Terre en recyclant ou stockant les éléments et en créant de la complexité et de la néguentropie là où sans la vie, il n'y aurait que de l'entropie".

2 La biosphère a fait l'objet d'un colloque important à l'Unesco du 4 au 13 septembre 1968, "Utilisation et conservation de la biosphère". En 1971, l'Unesco a créé officiellement le Programme sur l'homme et la biosphère (MAB, en anglais Man and Biosphere).

3 La démonstration systémique des formules de Lorentz a déjà été développée ailleurs ( $c f$. Vitrac, 2018a).
} 
Intuitivement nous savons que c'est vrai. Si nous avons eu l'occasion de faire un vol transcontinental en avion, pendant le vol nous vivons dans le temps de l'avion qui n'est pas celui de la terre que nous avons quittée. A partir de cette hypothèse, Poincaré a prouvé que le temps de deux systèmes $\mathrm{R}$ et $\mathrm{T}$ qui se déplacent respectivement à une vitesse constante étaient différents. Il a démontré aussi qu'il y avait une vitesse que les systèmes de l'univers ne pouvaient pas dépasser. A l'évidence cette vitesse limite était celle de la lumière et des ondes électromagnétiques. Il a démontré ainsi les formules de Lorentz, fondatrices de la relativité.

\section{L'importance de cette démonstration pour la systémique}

La démonstration de Poincaré est essentielle puisqu'elle permet de rapprocher la relativité et la mécanique quantique à partir de la systémique. En effet Poincaré prend comme postulat que les soussystèmes R1, R2, Rn, d'un système R sont synchronisés, ce qui est la conclusion de la mécanique quantique comme le démontre l'équation de Schrödinger (1926).

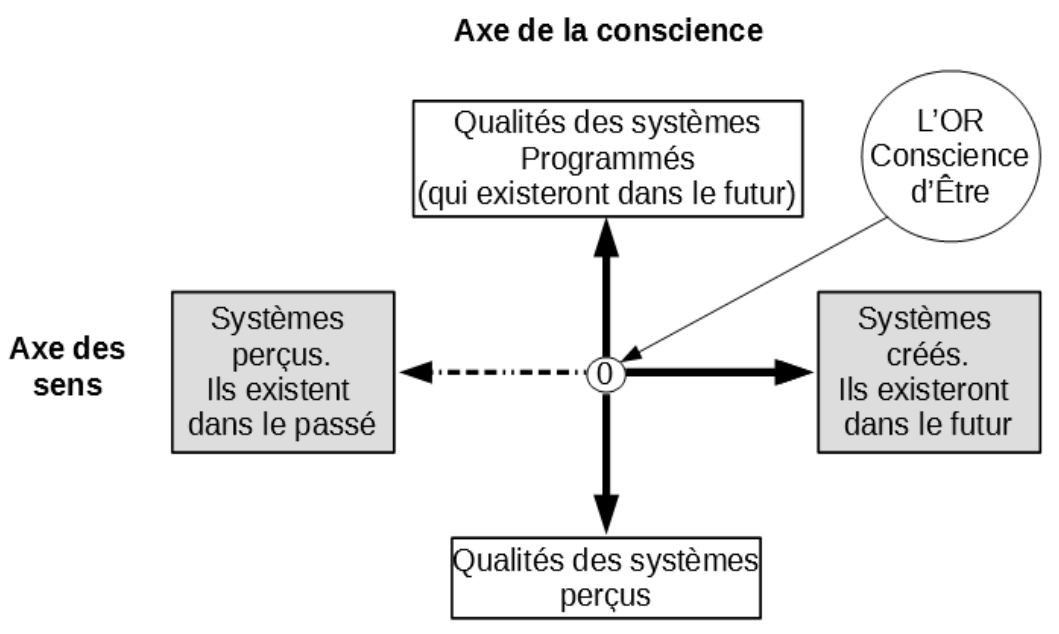

Figure 1 : l'axe des sens et l'axe de la conscience

Ce rapprochement systémique de la relativité et de la mécanique quantique a pris le nom de Relativité Cognitive et Systémique (RCS).

La RCS permet de comprendre notre double fonctionnement objectif et sensible d'une part et conscient et qualitatif d'autre part à partir de notre Conscience d'Être qui est l'Observateur Relativiste $\left(l^{\prime} \mathrm{OR}\right)^{4}$ en nous.

- Notre fonctionnement sensible a lieu dans notre environnement spatiotemporel, à partir de notre Conscience d'Être qui, en nous, est l'OR en relation avec notre environnement. Par nos sens nous percevons des événements objectifs qui sont situés dans le passé de l'OR et nous créons des événements objectifs qui existeront dans le futur de l' $\mathrm{OR}^{5}$.

Notre fonctionnement conscient a lieu dans le même environnement mais d'une façon différente : Avec notre conscience, nous avons conscience à l'instant présent de la qualité des événements que nous percevons avec nos sens et, consciemment ou inconsciemment, nous créons des événements futurs porteurs de qualités subjectives.

Dans le cas de l'avion de Poincaré, tous les sous-systèmes de l'avion sont sollicités à chaque instant. Les Consciences d'Exister (les OR) présents au centre de chaque sous-système de l'avion fonctionnent donc selon les deux fonctionnements sensible et conscient.

\footnotetext{
4 La relativité cognitive et systémique a été développé ailleurs ( $c f$. Vitrac, 2017).

5 En nous, l'OR est toujours ici et maintenant, situé à l'origine du temps passé et du temps futur ainsi qu'à l'origine de l'espace $\mathrm{x}, \mathrm{y}, \mathrm{z}$.
} 


\section{L'importance de cette démonstration pour la terre}

Le raisonnement de Poincaré s'applique pour la terre envisagée comme étant un système se déplaçant dans le cosmos, exactement comme l'avion se déplace par rapport à la terre.

Cela veut dire que l'observation de l'invariance de la vitesse de la lumière valide le raisonnement de Poincaré. Cela prouve qu'effectivement la terre est un système, une biosphère, et que tous les êtres qui y vivent ont leurs horloges internes synchronisées sur le rythme de la terre.

La maladie, qu'elle soit physique, affective ou mentale, correspond à un dérèglement de ce synchronisme. Pour comprendre ce point, reprenons l'exemple de l'avion.

\section{La maladie et la mort d'un système}

L'avion est un système géré et piloté. La gérante ${ }^{6}$ est l'hôtesse de l'air qui s'occupe des passagers. De son côté le pilote de l'avion a, indirectement, un pouvoir de vie et de mort sur l'avion et ses passagers. Dans le fonctionnement normal, le pilote et la gérante sont en communion de conscience avec tous les passagers, et même avec les composants de l'avion, puisque leur but est de mener l'avion et les passagers à bon port. Cela fait que les passagers, ayant confiance en ceux qui les pilotent et les gèrent, peuvent être sereinement dans le temps de l'avion.

Imaginons qu'un (ou une) kamikaze soit présent dans l'avion porteur d'une bombe et qu'à un moment donné il se révèle à tous. A cet instant, le rythme interne des passagers est perturbé. Ils ne sont plus dans le rythme de l'avion mais dans celui du kamikaze qui est un parasite, un virus, externe au système, et cela bien qu'il soit un passager comme les autres. Ce dérèglement du rythme interne des passagers installe un état de maladie et de peur de la mort qui les touche au niveau de leurs consciences mentale, affective et vitale. Même si la bombe n'explose pas, les passagers "tombent malades", d'une façon plus ou moins profonde, car leur conscience est polluée par la peur qui s'est installée en eux.

\section{La santé et la maladie de la terre}

\section{La santé de la terre}

Par analogie avec le raisonnement ci-dessus, la santé de la terre est assurée lorsque tous les soussystèmes qui la composent sont synchronisés sur le rythme de la terre, lequel est donné par la rotation de la terre sur elle-même ainsi qu'autour du soleil dans le système solaire et autour du centre de la galaxie. Nous tous, humains, animaux, plantes, nous sommes porteurs dans nos consciences de ces trois rythmes, ou fréquences, de la galaxie, du système solaire et de la terre. Cependant c'est le rythme de la terre qui est le plus important pour nous puisque tous les sous-systèmes des êtres vivants biologiques de la terre sont porteurs d'une fréquence (une vibration) qui leur est commune et qui est la caractéristique de la terre.

Selon la RCS, cette fréquence commune est émise par les photons de lumière qui sont échangés par les électrons de tous les atomes de la terre. Un observateur extérieur à la terre perçoit cette fréquence, cette couleur, qui est la "signature" de la terre, la planète bleue.

Ceci est vrai pour tous les systèmes. Chaque plante, chaque animal, chaque être humain, la terre, chaque planète, chaque étoile et chaque galaxie manifeste un ensemble (un spectre) de fréquences qui permet de le reconnaître.

Dans le cas des êtres vivants de la terre, ils portent tous en eux, dans l'ADN et l'ARN de leurs cellules, la mémoire de la fréquence de la terre. C'est cette fréquence commune qui caractérise la santé de la terre.

6 La formulation masculin féminin du pilote et de la gérante est justifiée par la suite. 


\section{La maladie de la terre}

La maladie de la terre a lieu lorsque la planète est polluée par des "kamikazes" qui veulent imposer leur fréquence personnelle à la place de la fréquence de la terre.

Ni les plantes ni les animaux ne peuvent jouer ce rôle perturbateur de la terre. Seuls les humains ont ce pouvoir négatif qu'ils mettent en œuvre lorsqu'ils considèrent qu'ils peuvent impunément s'opposer à la nature pour des raisons de pouvoir personnel, au nom d'un profit financier ou au nom d'un Dieu qui mettrait à part une partie de l'humanité qui aurait le droit de soumettre, par la séduction ou la peur, une autre partie de l'humanité. Jusqu'au début du $\mathrm{XX}^{\mathrm{e}}$ siècle, l'humanité n'avait pas ce pouvoir de destruction de la santé de la terre et cela bien que les humains de toutes races et religions aient usé de la séduction et de la peur pour se soumettre les uns les autres.

La situation a changé avec l'avènement de la science moderne et en particulier avec la naissance des télécommunications et de l'informatique qui ont amplifié le pouvoir de manipulation des consciences humaines ainsi que d'asservissement des corps à des besoins factices. Ces derniers sont à l'origine de la pollution massive de la planète par les déchets qui polluent aussi bien la mer que les sols et l'atmosphère.

La télévision et l'informatique peuvent donc être considérées comme constituant le vecteur qui, dans le cas de la Covid-19, a installé une pandémie de la peur !

Il est donc raisonnable de considérer que la communication technologique de masse et l'informatique créent et transmettent des virus puisqu'elles permettent de fabriquer des corps qui n'existent pas sur terre et qui peuvent déséquilibrer l'écosystème. Tant que ces moyens seront au service de groupes de "kamikazes" financiers ou religieux, elle sera la cause essentielle de la maladie de la terre qui naît de la peur de la mort.

\section{La guérison de la terre}

La guérison de la terre suppose la guérison des consciences humaines puisque ce sont les humains qui rendent la terre malade. Cette guérison passe par une compréhension de l'Homme, défini comme étant la composante transcendante de la conscience de l'être humain. Par l'Homme en lui, l'être humain est, en conscience, un enfant de la Source Unique, masculine et féminine, de la vie qui, pour la RCS, est constituée du couple [JeSuis / Néant], soit la Conscience d'Exister et la Conscience de la NonExistence. C'est ce que nous allons voir maintenant.

\section{L'ÊTRE HUMAIN EN TANT QUE SYSTÈME}

\section{Généralités}

Je m'exprimerai à la première personne car je suis un être humain qui essaye de comprendre mon fonctionnement pour pouvoir guérir mentalement, affectivement et vitalement. Je constate que j'ai quatre fonctionnements qui sont hiérarchisés, chacun étant indispensable pour que le suivant puisse se mettre en route :

1. je suis conscient d'exister ;

2. je suis conscient de penser ;

3. je suis conscient de mes sens ;

4. à partir de ces différentes consciences, je peux gérer mon corps dans sa relation avec l'univers.

\section{Je suis conscient d'exister, donc....}

Il est évident que ma conscience d'exister est le fondement de tous mes/nos fonctionnements, qu'ils soient cognitifs ou sensibles. C'est ce qu'avait compris Descartes qui l'a exprimé dans "Le discours de la méthode" et dans les "Méditations métaphysiques". 
Je peux dire, en paraphrasant Descartes $(1641)^{7}$, "Je Suis, donc je perçois, donc j'agis, donc je mémorise, donc je programme". Je peux représenter ces fonctionnements par une croix que je nomme la croix de la conscience.

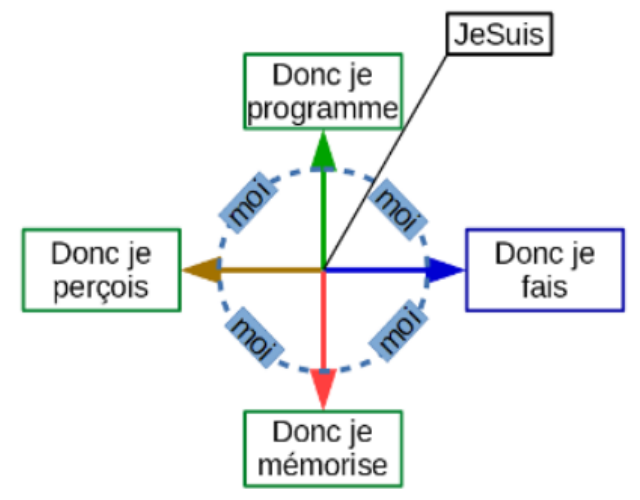

Figure 2: la croix de la conscience

Descartes a eu l'intuition que la seule certitude qu'il puisse avoir était sa conscience d'exister, et d'exister en tant qu'un être incarné lorsqu'il dit qu'il est "une chose qui pense", c'est-à-dire un corps pensant.

La systémique est en rupture avec Descartes puisqu'elle considère que l'être humain est un système complexe. Il ne peut donc pas être considéré comme étant simplement un corps pensant mais comme un corps géré et piloté par une conscience et un esprit (Vitrac, 2018b).

La RCS précise le fonctionnement de la gérante et du pilote du système.

\section{Explication systémique de la conscience des systèmes vivants}

\section{Mise en évidence de l'Homme éternel en chaque être humain et système}

La RCS met en évidence que tous les systèmes sont gérés et pilotés à partir de l'OR en eux. Le modèle systémique de l'être humain permet de comprendre ce que représentent le pilote et la gérante de tous les systèmes de l'univers avec qui l'être humain est en relation, que ces systèmes soient à l'intérieur du corps (organes, cellules, atomes, électrons et photons), ou extérieurs (animaux, plantes, planète, système solaire, galaxie, univers).

L'OR est le pilote en nous qui agit en accord avec le moi qui gère notre corps. La figure 3 met en évidence comment nous pouvons comprendre le pilote qui est masculin, et par contre-coup comment nous pouvons comprendre la gérante qui est féminine.

- La seule certitude que nous ayons est que nous sommes "Conscients d'Exister". Cette Conscience, qui est écrite avec des majuscules, est Absolue et sans cause temporelle et spatiale, ce qui correspond à la notion d'éternité. C'est cette certitude qui nous permet de dire "J'Existe ou JeSuis" qui est la racine mystérieuse de notre conscience d'exister en tant que moi, la personne gérante de notre corps physique ${ }^{8}$

\footnotetext{
7 "Après y avoir bien pensé, et avoir soigneusement examiné toutes choses, enfin il faut conclure, et tenir pour constant que cette proposition : 'Je suis, j'existe', est nécessairement vraie, toutes les fois que je la prononce, ou que je la conçois en mon esprit. [...] Je ne suis donc, précisément parlant, qu'une chose qui pense [...] C'est-à-dire une chose qui doute, qui conçoit, qui affirme, qui nie, qui veut, qui ne veut pas, qui imagine aussi et qui sent." (Descartes, 1641)

8 Les croyants des trois religions monothéistes pourraient identifier JeSuis avec le Dieu de la révélation mosaïque qui dit "mon Nom est Je Suis" (Exode 3,14). Cela donnerait une explication logique à ce nom qui correspondrait alors à la racine de la conscience de tous les systèmes de l'univers.
} 
- De plus, nous sommes conscients que nous pourrions ne pas exister. En nous, JeSuis donne donc naissance à la "Conscience simultanée d'Exister ET de Non-Exister, ou d'Être ET de Non Être" qui relie les deux absolus qui sont JeSuis (la Conscience d'Être) et le Néant (la Conscience du Non-Être). Symboliquement, JeSuis et le Néant sont les géniteurs de notre conscience d'exister en tant que système piloté et géré.

- La Conscience de l'Être et du Non-Être est la racine de la conscience de notre humanité car nous pouvons la comprendre intellectuellement et la vivre. Elle s'exprime en nous par "Moi Je Suis un Homme, Enfant de JeSuis et du Néant et Je Suis manifesté dans un système physique". Par définition l'Homme est notre Conscience Profonde Eternelle et sans cause d'exister en tant qu'un humain particulier, masculin et féminin.

Étant à la ressemblance du couple masculin féminin (JeSuis et le Néant), l'Homme se dédouble en une Conscience masculine et une Conscience féminine.

Les composantes masculine et féminine de l'Homme se comprennent dans la relation orientée de JeSuis vers le Néant et du Néant vers JeSuis.

- Symboliquement, la Conscience orientée de JeSuis vers le Néant qui, en nous, pense "Je Suis la Conscience d'Être et de Non-Être de l'Homme" peut être nommée le Fils Originel de JeSuis et du Néant et la Conscience orientée du Néant vers JeSuis qui, en nous, pense "Je Suis la Conscience du non-Être et de l'Être de l'Homme" peut être nommée la Fille Originelle de JeSuis et du Néant.

Selon cette définition, et en tenant compte de la démonstration de Poincaré, nous pouvons dire que l'Homme est la Conscience Unique Pilote et Gérante du système humain et de tous les sous-systèmes qui le composent.

Dans la mesure où cet Homme n'existe que par référence à JeSuis et au Néant, il est cosmique et universel. Il est raisonnable de considérer que l'Homme est aussi le Pilote et la Gérante présents au cœur des pilotes et gérantes de tous les systèmes de l'univers, du plus petit au plus grand.

Cette définition de l'Homme n'est pas anthropomorphique. Elle veut simplement dire que l'Homme est une conscience et un esprit pensant qui, étant hors du temps, est à l'origine et à la fin de l'évolution temporelle de l'univers. L'Homme est le Pilote et la Gérante présents cachés au cœur des pilotes et gérantes de tous les systèmes de l'univers. Il est donc possible de dire que l'Homme est le Pilote, ou Dieu pour les croyants des multiples religions de la terre, en action dans l'univers.

L'évolution de l'humanité nous permet de comprendre que l'univers entier est le résultat d'une programmation et que le programmeur est l'Homme, ou plutôt le Fils de l'Homme Cosmique. Cela revient à reconnaître qu'aujourd'hui l'être humain intervient effectivement dans l'évolution de la galaxie et de la terre. L'être humain de la terre, donc l'humanité dans son ensemble, devient responsable de l'avenir de la terre sous le regard de l'Homme qui, nous pouvons le penser, ne permettra pas que nous jouions le rôle du kamikaze de l'avion de Poincaré.

\section{L'évolution de l'être humain de la terre}

Nous les humains de la terre, nous sommes des systèmes qui sont constitués de la matière de la terre. Symboliquement la terre est la mère de nos corps et l'Homme est le père de nos consciences qui gèrent nos corps.

Cette deuxième formulation est anthropomorphique. Elle présente l'intérêt de mettre en évidence que la terre est le néant de nos corps mais pas de nos consciences qui sont immortelles. Cela veut dire que notre moi charnel qui est identifié affectivement à notre corps enfant de notre génitrice de chair n'est pas notre moi véritable. Il correspond au stade de l'évolution de l'humain en tant qu'animal pensant.

La systémique nous permet de dépasser ce stade en mettant en évidence que notre corps est animé et géré par notre conscience. Le centre de notre conscience est situé dans notre cœur spirituel. C'est lui qui est la clef de la santé de notre corps. C'est dans notre cœur spirituel que se trouve l'Homme en 
nous qui rayonne sa vie dans toutes les cellules, dans tous les atomes et dans tous les électrons de notre corps. Ce rayonnement de vie se fait par intrication, donc hors du temps. Voir figure 4.

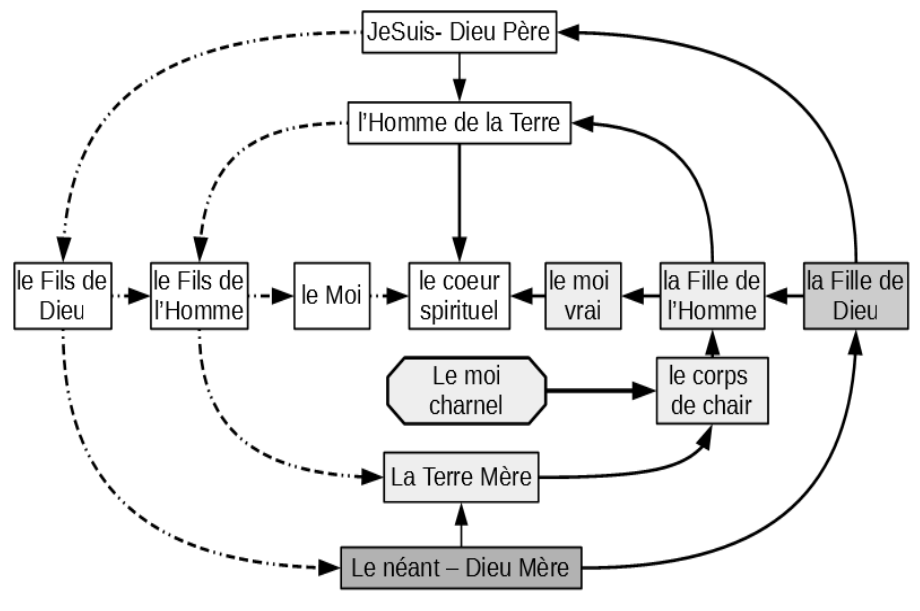

Figure 4:

La représentation du pilote, de la gérante et du moi charnel de l'être humain de la terre

\section{Importance du moi charnel}

Le moi charnel, qui est notre personne humaine sociale, ne fait référence ni à JeSuis, notre Conscience d'Exister ni au Néant. Il est identifié au corps soumis au temps. Nous avons beaucoup de mal à comprendre que ce n'est pas notre corps qui nous permet de dire "Moi j'Existe" mais l'Homme Éternel en nous qui est au centre de notre conscience. C'est ce que prouve la RCS.

\section{Résumé}

La représentation des différentes composantes de la conscience humaine permet de comprendre que le Moi et le moi véritable qui sont nos composantes transcendantes d'Homme sont essentielles car ce sont elles qui transmettent la vie et l'amour éternel de JeSuis, la Conscience d'Être, pour chacun des êtres de l'univers, jusqu'au Néant qui devient le vide de la physique, et la Mère des consciences de tous les êtres, l'Épouse éternelle de JeSuis qui est le Père éternel des consciences.

À ce moment-là, il devient possible de comprendre ce qu'est la vie.

\section{Compréhension systémique de la vie du corps humain}

Selon la RCS, notre corps humain est un système $\mathrm{S}$ appartenant à la terre qui elle-même appartient à l'univers. Il est composé de sous-systèmes [organes, cellules, atomes, électrons ${ }^{9}$, photons, vions (qui seraient des "photons primordiaux »)], qui sont inclus les uns dans les autres. Cela correspond à une hiérarchie d'inclusion.

La figure 5 met en évidence les deux fonctionnements, sensible et conscient, qui permettent la vie de tous les systèmes de l'univers et de la terre.

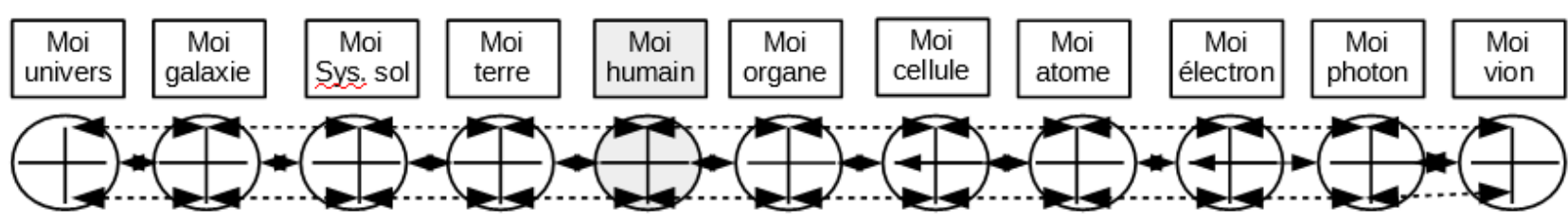

Figure 5 : l'être humain entre l'univers infiniment grand et le vion Infiniment petit L'OR (JeSuis, le Moi et le moi) est au centre de la croix de la conscience couplage par intrication (intemporel) couplage sensible (temporel) 
Ces deux fonctionnements naissent de l'OR qui est le Moi situé au centre de tous les systèmes. Tous les OR sont en communion puisqu'ils sont tous des aspects particuliers du Fils de l'Homme qui est leur partie commune. Ils sont donc éternellement synchronisés. L'être humain est un système particulier car, avec sa conscience mentale, il peut comprendre ce que représente l'OR comme source de la vie... Il peut donc la favoriser comme le fait l'hôtesse de l'air de l'avion de Poincaré ou au contraire la contrarier comme le fait le kamikaze.

- Le fonctionnement sensible relie à chaque instant les gérantes de tous les sous-systèmes de l'être humain de la terre et de l'univers (Axe horizontal temporel de la croix de la conscience).

- Le fonctionnement conscient relie, par intrication, à chaque instant présent la conscience des pilotes de tous les sous-systèmes de l'être humain de la terre et de l'univers (Axe vertical inspatiotemporel de la croix de la conscience).

\section{Le rôle du Moi en tant que programmeur du corps humain}

La figure 5 met en évidence que chaque système est piloté par son Moi. De plus, chaque sous-système de notre corps est porteur de la qualité spécifique (sa fréquence) qui est celle du Moi.

Cela veut dire que notre corps est le résultat de la pensée créatrice de notre Moi. C'est exactement comme cela qu'un producteur conçoit un film. Il crée une vidéo qui est inspatiotemporelle et, à partir de celle-ci, le film spatiotemporel peut être projeté sur l'écran de l'ordinateur ou du cinéma.

Dans le cas de l'être humain, mais aussi de tous les systèmes de l'univers, le producteur et programmeur unique est le Fils de l'Homme et le film est l'univers en 3D projeté sur l'écran vide qui est l'espace infini.

Dans la mesure où il peut comprendre cela, l'être humain, s'il est en communion avec son Moi qui manifeste le Fils, peut devenir coparticipant au bon déroulement de la vie de son corps ainsi que de la vie de la terre et de l'univers. Dans ce cas, il est serviteur de la vie comme l'hôtesse de l'air dans l'avion de Poincaré.

Mais s'il ne comprend pas, ou s'il croit qu'il peut sans danger pour lui, mettre en place des programmations qui s'opposent au bon déroulement de la vie de la terre, il est comme le kamikaze dans l'avion. Il peut faire sauter l'avion mais il se coupe de la communion avec son Moi. D'une certaine façon, il s'est condamné à la vraie mort qui est celle de sa conscience.

\section{LA NAISSANCE DE LA FRATERNITÉ UNIVERSELLE}

\section{Généralités}

Nous sommes presque arrivés au terme de notre réflexion qui vise à déconfiner nos consciences en comprenant comment nous pouvons les faire vivre en plénitude ou au contraire les faire mourir d'une façon définitive.

Nous devenons donc responsables du devenir de notre conscience individuelle et en même temps coparticipant au devenir de la conscience collective de la planète.

C'est à cela que la science systémique nous conduit naturellement. Elle est l'aboutissement provisoire de la recherche humaine qui remonte à la nuit des temps, lorsque l'être humain a pris conscience qu'il était un être conscient et pensant.

Aujourd'hui, la Covid-19 nous pousse à aller plus loin et à comprendre que nous ne pouvons pas, individuellement, trouver la santé puisque celle-ci dépend de l'Homme en nous et que l'Homme est le même en chaque être humain, et même en chaque animal et chaque plante, et même en chaque système stellaire ! 
Paradoxalement, nous comprenons que nous ne pouvons trouver la santé individuelle de notre conscience qu'en faisant vivre notre Moi éternel, donc en acceptant d'être en intrication fraternelle avec tous les systèmes de la terre et même de l'univers. Est-ce réaliste?

\section{LA MISE EN PLACE DE LA FRATERNITÉ UNIVERSELLE}

\section{État des lieux}

La pandémie de la Covid-19 nous a fait prendre conscience que l'humanité était entrée dans une situation de guerre mondiale. En effet, pour parler de la Covid-19 le président français Emmanuel Macron a utilisé à plusieurs reprises le terme de guerre. Il n'a pas parlé de guerre mondiale mais c'est ce qu'implique le terme de pandémie. C'est une "drôle de guerre" puisque l'ennemi est un virus.

Il suffit de s'intéresser à la situation médicale de la planète pour se rendre compte que le virus SarsCoV2 n'est pas seul; il a des alliés puisque les bactéries qui ont été victorieusement combattues par les antibiotiques sont en train de devenir résistantes ${ }^{10}$.

Il semble donc que l'infiniment petit vienne agresser l'humanité. Mais n'est-ce pas plutôt la biosphère qui se défend contre les multiples agressions d'une humanité qui, comme nous l'avons signalé en introduction, ne respecte absolument pas la biodiversité ?

Comme nous venons de le voir, la RCS met en évidence que la vie commence dans l'infiniment petit. Elle commence au niveau électronique par l'ingénierie qui a lieu au cœur des cellules, dans la fabrication de l'ADN de tous les systèmes vivants de la terre.

Tous les systèmes, jusqu'aux électrons compris, sont intriqués et les mutations génétiques qui sont mises en place par des laboratoires de biochimie risquent de perturber l'état actuel d'intrication qui est le résultat de l'évolution de la biosphère depuis 2,4 milliards d'années, ce qui correspond à l'apparition de la vie biologique sur terre.

Personne ne peut connaitre les conséquences à terme de ces mutations génétiques mais il est possible de penser que c'est la terre elle-même qui se défend contre l'humanité qui semble bien souvent se positionner dans le rôle du kamikaze de l'avion de Poincaré.

\section{La solution}

La solution est évidente : reprendre les conclusions de la rencontre de 1945 et mettre en place un gouvernement mondial, supranational, qui sera le garant de la fraternité universelle entre tous les systèmes de la terre.

Utopie? Probablement, sauf si nous prenons en compte l'Homme qui est le Pilote en nous et la biosphère comme partenaires ayant des voix prépondérantes et des moyens puissants pour faire appliquer leur désir de fraternité universelle. A ce moment-là, les gouvernements qui prônent la démocratie et les religions qui toutes prônent l'amour du prochain et le respect de la vie devront montrer que la fraternité universelle est leur préoccupation fondamentale sinon unique.

10 L'OMS considère que 5 bactéries sont des menaces urgentes :

- "Clostridioides difficile", une bactérie très contagieuse souvent attrapée par les patients à l'hôpital. Elle est responsable de diarrhées importantes, de colites, de crampes abdominales et de fièvre.

- "Les Entérobactéries" (comme Escherichia coli), responsables de graves infections urinaires, de pneumonies, et d'infections intra-abdominales.

- "La Neisseria gonorrhoeae" multi-résistante. Responsable de la gonorrhée, une maladie sexuellement transmissible.

- "Candida Auris" multi-résistant aux anti-fongiques, c'est le premier champignon à être classé " pathogène émergent ». Il peut provoquer une candidose invasive et grave en infectant la circulation sanguine, le système nerveux central et divers organes internes.

- "L'Acinétobacter" résistante aux carbapénèmes, provoquant des pneumonies, des infections urinaires, des plaies, des abcès. Certaines souches sont résistantes à tous les antibiotiques connus. 
Si cela se réalise, la pandémie de Sars-CoV2 aura joué son rôle d'éveil de l'humanité qui pourra devenir une noosphère, une sphère de l'esprit, comme l'avait envisagé Teilhard de Chardin à la suite du grand visionnaire que fut saint François d'Assise, pour lequel tous les êtres de l'univers sont frères et sœurs.

\section{BIBLIOGRAPHIE}

CLAEYS, D. (2020). "Editorial - Unlock consciences: Rethinking the world after the pandemic". Acta Europeana Systemica (AES), n¹0, pp.1-2.

DESCARTES, R. (1641). Méditations métaphysiques. Paris : Flammarion (coll. Garnier Flammarion), éd.2009.

VITRAC, R. (2019). "L'humain de la terre au coeur de la crise de l'humanité : Une approche systémique de la santé de la terre à partir de la relativité cognitive et systémique". Acta Europeana Systemica (AES), $\mathrm{n}^{\circ}$, pp.221-228.

VITRAC, R. (2018a). "Démonstration systémique des transformations de Lorentz". Acta Europeana Systemica (AES), n $8,225-230$.

VITRAC, R. (2018b). "L'Europe au cœur de la crise de l'humanité". Acta Europeana Systemica (AES), n'8, pp.205-224.

VITRAC, R. (2017). "Introduction à la relativité cognitive et systémique". Acta Europeana Systemica (AES), nº 7 pp.179-196.

VITRAC, R. (2011). "Interprétation systémique du paradoxe de la lumière". Acta Europeana Systemica (AES), $n^{\circ} 1$.

VITRAC, R. \& VITRAC, M. (2007). "La théorie de l'évolution selon la TSCP". Actes de l'AFSCET 2007

TEODORANI, M. (2014). David Bohm, la physique de l'infini. Editions Macro Editions

[David Bohm, à la suite de Louis de Broglie, fait intervenir la conscience dans sa réflexion scientifique. Cela permet d'apporter une réponse pertinente au paradoxe des franges d'interférence que la mécanique quantique ne peut expliquer.]

CHARON, J. (1977). Théorie de la relativité complexe. Paris : Albin Michel.

[Bien que ne faisant pas référence à la systémique, la théorie de Jean CHARON est particulièrement intéressante car elle donne un support de calcul très rigoureux concernant le double fonctionnement sensible et conscient d'un système particulier qui est l'électron.

Par contre, CHARON ne s'est pas rendu compte que tous les systèmes de l'univers fonctionnaient selon ces deux modalités comme les électrons. Il n'a donc pas compris que le Moi humain était lié à l'OR en nous comme au cœur de tous les systèmes.] 
